Article

\title{
Reduced-Order Modeling for and Vibration Characteristics Analysis of a Hard-Coated Mistuned Blisk
}

\author{
Feng Gao (10) and Wei Sun * \\ School of Mechanical Engineering \& Automation, Northeastern University, Shenyang 110819, China; \\ peakgaoneu@163.com \\ * Correspondence: weisun@mail.neu.edu.cn
}

Received: 16 June 2017; Accepted: 12 July 2017; Published: 18 July 2017

\begin{abstract}
This paper develops a damping strategy for the vibration reduction of a mistuned bladed disk (blisk) by depositing hard coating on its blades, and systematically investigates the vibration characteristics of the hard-coated mistuned (HCM) blisk. By using an improved fixed-interface component modal synthesis method (fixed-ICMSM), a reduced-order model (ROM) of the HCM blisk is established. Then, based on the proposed ROM, solutions of eigenvalue equations are carried out to obtain the natural frequencies and mode shapes. Further, modal loss factors and a damping matrix of the HCM blisk are achieved by taking advantage of the modal strain energy method and the proportional damping model, respectively. Moreover, the frequency response function of the HCM blisk, which can exhibit dynamic behaviors, was deduced. Finally, a mistuned blisk with a deposited NiCoCrAlY + YSZ hard coating on both sides of the blades is chosen as a study case to conduct a finite element analysis, and the results are compared with those obtained from the experimental test in terms of natural frequencies and mode shapes. The variation of the natural frequencies, the modal loss factors, and the frequency response function generated by the NiCoCrAlY + YSZ hard coating are studied, and the influence of coating area on damping capacity is further discussed.
\end{abstract}

Keywords: mistuned blisk; hard coating; reduced-order modeling; vibration characteristics; damping capacity

\section{Introduction}

Due to the superior improvement of aerodynamic performance and structural design along with weight reduction, the application of bladed disks (blisks) is widely increasing in aero-engines. A blisk is composed of a single-piece component in place of the conventional assembly of a disk and detached blades. In fact, in contrast with bladed disk assemblies, the single-piece-made structures bring about a significant problem in that the internal damping of the blisk decreases generally and enormously without a dovetail attachment root and friction interfaces, which can dissipate the vibratory energy straightforwardly [1]. As a result, blisks tend to be subject to high vibration levels and severe resonances in the worst cases, which always give rise to a risk of high cycle fatigue and even failure. Thus, in particular, the exploitation of additional damping technology is very necessary for the vibration control of a blisk.

In its engineering applications, hard coating has been studied and employed widely by many researchers to improve surface performance, such as the wear resistance (or anti-friction resistance) generated by the increase of hardness [2-4], the increase of fatigue strength related to residual stresses [5], high-temperature resistance [6], and anti-corrosion [7] with nicer stability. In recent years, the damping effect of hard coating, generated by the internal particle friction of coating material as illustrated in Figure 1 [8], was found and investigated gradually by many researchers [9-11], 
and has been used as the damping treatment for vibration control with considerable prospects. By making use of the Lindstedt-Poincaré perturbation method, the vibration characteristics of a hard coating-laminated plate, in which anisotropic hard coating was deposited on a single side of isotropic metal, were investigated by Yang et al. [12], and compared with the results obtained by the finite element method and experimental tests, respectively. By taking advantage of the semi-analytical method based on polynomial fitting, the vibration response and resonant frequencies analysis of a cantilever plate coated with a nonlinear $\mathrm{MgO}+\mathrm{Al}_{2} \mathrm{O}_{3}$ hard coating were carried out accurately by Sun et al. [13], and compared with an experiment and a linear calculation, respectively. Subsequently, Sun et al. [14] conducted a free vibration analysis of a hard-coated cantilever cylindrical shell on the basis of Love's theory and the Rayleigh-Ritz method, and compared with an analytical calculation and an experiment, respectively, with respect to natural frequencies. In 2016, Sun and Liu [15] studied the modeling and vibration of a composite beam coated with a strain-dependent hard coating, and the vibration responses at resonance frequencies of the cantilever beam were calculated and compared with an experiment and a linear calculation, respectively. Moreover, Sun and Kari [16] developed an increase-damping method by depositing coating on compressor blades, and found that hard coating contributed to a decrease in the peak stress of the compressor blades, and therefore improved its life time. Based on all of the above, a damping strategy for the vibration reduction of a mistuned blisk by depositing hard coating on its blades is proposed, and then investigated systematically in this paper.

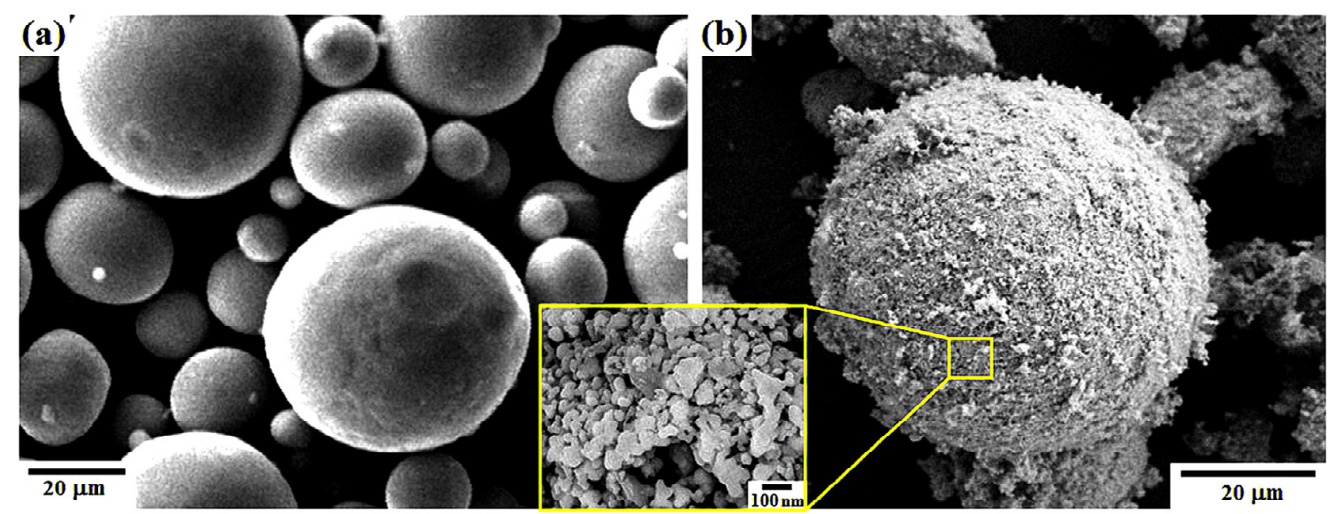

Figure 1. Surface SEM image of (a) NiCoCrAlY powders; (b) agglomerated nano-YSZ powders [8]. (Copyright 2016 Elsevier).

In contrast with the lumped parameter model and the beam-plate model, which are usually employed to study fundamental mechanisms, finite element models (FEMs) of blisks that include millions of degrees of freedom (DOFs) are more and more extensively utilized for meeting the requirement of higher accuracy in industry applications. In the ideal, tuned conditions, the structural properties and material parameters of the sectors are assumed to be the same and identical, referred to as cyclic symmetry. Thus, the vibration characteristics of the tuned blisk can be solved efficiently by using a single piece of sector instead of the whole structure. However, small, random variations between sectors (blades generally), referred to as mistuning, are in existence in reality inevitably because of, among other things, the manufacturing tolerance, material deviation, and wear during the working process. Due to the absence of cyclic symmetry resulting from mistuning, the vibration characteristics of the mistuned blisk have to be analyzed by making full use of all sectors with a large amount of calculation. Moreover, given the limited computing resources, it is very difficult to carry out a finite element analysis (FEA) for the whole structure of the mistuned blisk directly.

In answer to this unavoidable issue, some kinds of model reduction technologies for the mistuned blisk have been proposed and improved upon by scholars. The first generation of finite element reduced-order models (FE ROMs) were established by using the component modal synthesis (CMS) method. Then, a novel FE ROM was developed by Yang and Griffin [17] in 2001 by using the 
relationship between the tuned and mistuned modes, called the subset of nominal modes (SNM) method. Subsequently, a simplified SNM method based on a single family of modes, called the fundamental mistuning model (FMM) method, was presented by Feiner and Griffin [18] in 2002. Lim et al. [19] proposed a component mode mistuning (CMM) method, in which the ROM of a mistuned blisk was generated by making use of both tuned system modes and blade component modes. CMS methods with systemic theory is a very effective and efficient way to solve vibration characteristics problems for large and complex mechanical structures. Its fundamental philosophy is breaking up the whole into parts and then gathering the parts into a whole. In general, depending on the boundary conditions, it consists of the fixed interface component modal synthesis method (fixed-ICMSM) proposed by Hurty in the 1960s [20,21], the free interface component modal synthesis method (free-ICMSM) proposed by Benfield and Hruda [22] in 1971, and the hybrid interface component modal synthesis method (hybrid-ICMSM) proposed by MacNeal [23] in 1971. For increasing the computational accuracy, the fixed-ICMSM method was further improved by Suarez [24] without taking orthonormal modes into account, but the improved method was no longer suitable for the large and complicated model. In 2014, Bai et al. [25] established a double ROM for mistuned blisks by using an improved hybrid-ICMSM with lower accuracy. In this paper, based on the dynamic stiffness on the component interface, an improved, common CMS method considering higher order modal truncations is proposed for the vibration analysis of HCM blisks.

The work described in this paper highlights reduced-order modeling and a vibration characteristics analysis for HCM blisks. Firstly, for establishing the rational ROM of the HCM blisk used in the vibration analysis, an improved component modal synthesis method (ICMSM) based on dynamic stiffness on component interface is developed by making use of a series of expansions and double coordinating conditions, including geometrical compatibility and force equilibrium. Then, on the basis of the proposed ROM, natural frequencies, mode shapes, and the modal loss factors of the HCM blisk are achieved by the eigenvalue equations and the modal strain energy (MSE) method. Moreover, the frequency response function of the HCM blisk is deduced by using the proportional damping model to obtain a damping matrix. Finally, a mistuned blisk with deposited NiCoCrAlY + YSZ hard coating on both sides of the blades is selected as a study case to carry out a numerical calculation, and the results are compared with those obtained from an experimental test by both natural frequencies and mode shapes. The variation trends of the vibration characteristics of the mistuned blisk generated by a $\mathrm{NiCoCrAlY}+$ YSZ hard coating are studied in terms of natural frequencies, modal loss factors, and a frequency response function, and the specific influence of coating area on the modal loss factors and the frequency response function of the mistuned blisk are further discussed.

\section{Theoretical Analysis of the HCM Blisk}

\subsection{Reduced-Order Modeling of the HCM Blisk Using ICMSM}

The solid model and FEM of the mistuned blisk and the HCM blisk are shown in Figure 2. The blades with red color and the rest with gray color are the mistuned and tuned structures, respectively. Moreover, hard coating damping, represented by the blue color, is deposited on both sides of all of the blades of the mistuned blisk. An ROM of the HCM blisk, regarded as a large and complex structure, is very essential and crucial to be constructed for the purpose of decreasing the amount of calculation without significantly altering computational accuracy. 


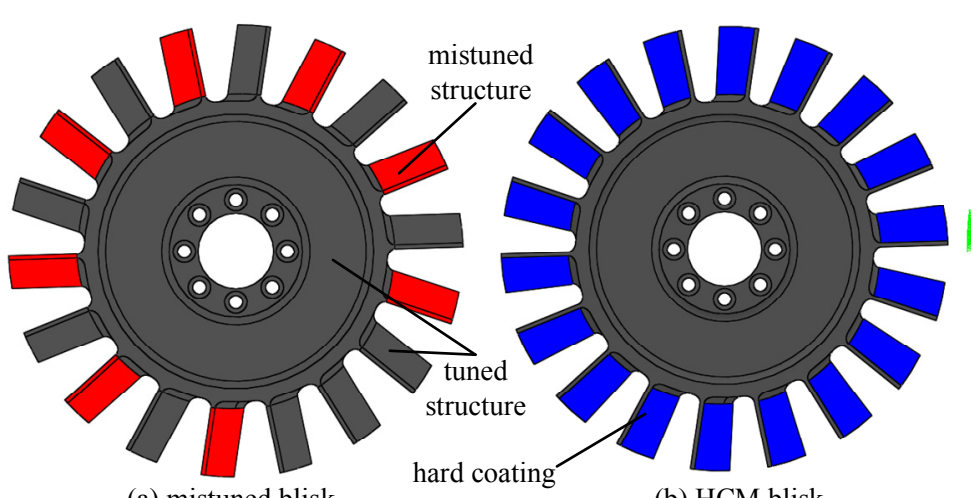

(a) mistuned blisk

(b) HCM blisk

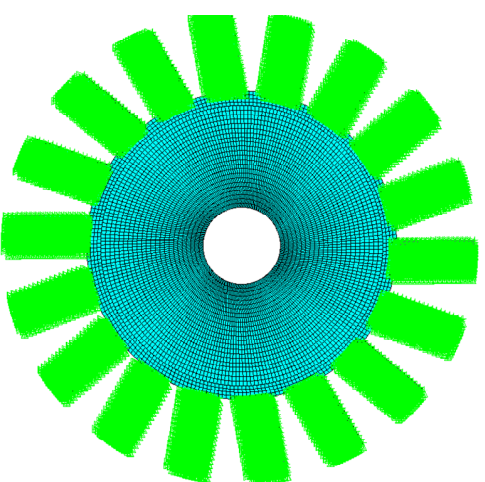

(c) HCM blisk of FEM

Figure 2. Solid model of (a) the mistuned blisk, (b) the hard coated mistuned (HCM) blisk and (c) FEM of the hard coated mistuned (HCM) blisk.

The basic procedure for reduced-order modeling of the HCM blisk based on the fundamental principle of the proposed ICMSM is illustrated in Figure 3. The specific equations of the ICMSM are derived and organized as follows: (a) derivation of the equation of dynamic stiffness on the component interface, (b) Taylor expansion of the equation of dynamic stiffness on the component interface, (c) modal synthesis of the HCM blisk based on the double coordinating conditions, and (d) elimination of the high derivative of the interface displacement equation.

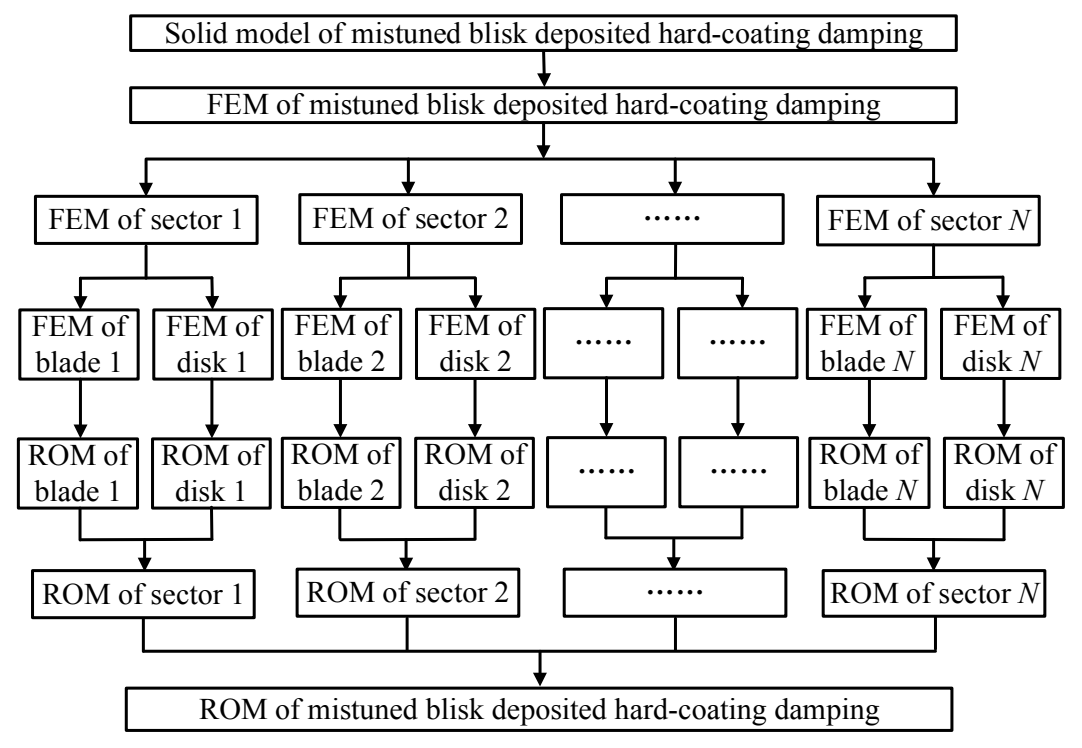

Figure 3. Fundamental principle of the improved component modal synthesis method (ICMSM) for the HCM blisk. ROM, reduced-order model.

The equation of motion of the HCM blisk without considering damping can be expressed as

$$
M_{\mathrm{cS}} \ddot{X}_{\mathrm{cs}}+K_{\mathrm{cs}} X_{\mathrm{cs}}=F_{\mathrm{cs}}
$$

where $K_{\mathrm{cs}}$ and $M_{\mathrm{cs}}$ refer to the stiffness and mass matrices of the HCM blisk, respectively, and $X_{\mathrm{cs}}$ and $F_{\mathrm{cs}}$ represent the column vector of the displacement and exciting force of the HCM blisk, respectively. 
Then, the stiffness and mass matrices and the displacement vector of the components can be written, respectively, as $K^{\mathrm{r}}, M^{\mathrm{r}}$ and $X^{\mathrm{r}}$ :

$$
\boldsymbol{K}^{\mathrm{r}}=\left[\begin{array}{cc}
\boldsymbol{K}_{i i}^{\mathrm{r}} & \boldsymbol{K}_{i j}^{\mathrm{r}} \\
\boldsymbol{K}_{j i}^{\mathrm{r}} & \boldsymbol{K}_{j j}^{\mathrm{r}}
\end{array}\right], \boldsymbol{M}^{\mathrm{r}}=\left[\begin{array}{ll}
\boldsymbol{M}_{i i}^{\mathrm{r}} & \boldsymbol{M}_{i j}^{\mathrm{r}} \\
\boldsymbol{M}_{j i}^{\mathrm{r}} & \boldsymbol{M}_{j j}^{\mathrm{r}}
\end{array}\right], \boldsymbol{X}^{\mathrm{r}}=\left\{\begin{array}{c}
\boldsymbol{X}_{i}^{\mathrm{r}} \\
\boldsymbol{X}_{j}^{\mathrm{r}}
\end{array}\right\}
$$

where, $r=b$ or $d$, and $b, d$ are the hard-coated blades and disk, and $i$ and $j$ refer to the internal and external DOF, respectively. In order to express the equations conveniently and succinctly, $K^{\mathrm{r}}, M^{\mathrm{r}}$ and $X^{\mathrm{r}}$ are simplified as $K, M, X$, respectively, in the following derived equations. For any one of the selected components of the HCM blisk, the equation of motion without considering damping can be expressed as

$$
M \ddot{X}+K X=f
$$

Or, the equation of motion can be rewritten as

$$
\left\{\left[\begin{array}{cc}
\boldsymbol{K}_{i i} & \boldsymbol{K}_{i j} \\
\boldsymbol{K}_{j i} & \boldsymbol{K}_{j j}
\end{array}\right]-\lambda\left[\begin{array}{cc}
\boldsymbol{M}_{i i} & \boldsymbol{M}_{i j} \\
\boldsymbol{M}_{j i} & \boldsymbol{M}_{j j}
\end{array}\right]\right\}\left\{\begin{array}{c}
\boldsymbol{X}_{i} \\
\boldsymbol{X}_{j}
\end{array}\right\}=\left\{\begin{array}{c}
\boldsymbol{0}_{i} \\
f_{j}
\end{array}\right\}
$$

where $f$ and $\lambda$ refer to the interface force and eigenvalues of the component, respectively. In the case of a fixed interface $\left(X_{j}=0\right)$, the vector of internal displacement $X_{i}$ can be given as below:

$$
\begin{gathered}
\boldsymbol{X}_{i}=\sum_{p=1}^{P} x_{i}^{p} \varphi_{i}^{p} \\
\left(\boldsymbol{K}_{i i}-\lambda \boldsymbol{M}_{i i}\right) \varphi_{i}=\mathbf{0}
\end{gathered}
$$

where $P$ and $x^{\mathrm{P}}$ represent the mode index and corresponding mode coordinate, respectively. In addition, the natural frequencies $\Lambda_{i i}$ and mode shapes $\phi_{i i}$ of the component can be obtained from Equations (5) and (6) as

$$
\begin{gathered}
\Lambda_{i i}=\operatorname{Diag}\left[\lambda_{1}, \cdots, \lambda_{k}, \lambda_{k+1}, \cdots, \lambda_{n}\right]=\operatorname{Diag}\left[\Lambda_{k k}, \Lambda_{h h}\right] \\
\Phi_{i i}=\left[\varphi_{1}, \cdots, \varphi_{k}, \varphi_{k+1}, \cdots, \varphi_{n}\right]=\left[\Phi_{i k}, \Phi_{i h}\right]
\end{gathered}
$$

Supposing I as the identity matrix, Equations (7) and (8) (or natural frequencies and mode shapes) are satisfied with the particular condition of orthogonal-normalization, as

$$
\begin{gathered}
\Phi_{i i}{ }^{T} \boldsymbol{M}_{i i} \Phi_{i i}=\boldsymbol{I}_{i i} \\
\Phi_{i i}{ }^{T} \boldsymbol{K}_{i i} \Phi_{i i}=\Lambda_{i i}
\end{gathered}
$$

Substituting Equations (9) and (10) into Equation (6), Equation (11) can be obtained as below:

$$
\boldsymbol{K}_{i i}-\lambda \boldsymbol{M}_{i i}=\Phi_{i i}{ }^{-T}\left(\Lambda_{i i}-\lambda \boldsymbol{I}_{i i}\right) \Phi_{i i}{ }^{-1}=\Phi_{i i}{ }^{-T} \Delta(\lambda) \Phi_{i i}{ }^{-1}
$$

By using the inverse matrix of Equation (11), the dynamic flexibility equation of the internal DOF can be achieved as

$$
\boldsymbol{D}_{i i}{ }^{-1}(\lambda)=\left(\mathrm{K}_{i i}-\lambda \boldsymbol{M}_{i i}\right)^{-1}=\Phi_{i i} \Delta_{i i}{ }^{-1}(\lambda) \Phi_{i i}{ }^{T}
$$

From the first line of Equation (4), the relationship between the internal and external displacement can be obtained as

$$
\boldsymbol{X}_{i}=-\left(\boldsymbol{K}_{i i}-\lambda \boldsymbol{M}_{i i}\right)^{-1}\left(\boldsymbol{K}_{i j}-\lambda \boldsymbol{M}_{i j}\right) \boldsymbol{X}_{j}
$$


Then, from the second line of Equation (4), the relationship between the interface force and displacement is deduced as

$$
f_{j}(\lambda)=D_{j j}^{*}(\lambda) X_{j}
$$

where $D_{j j}^{*}(\lambda)$ refers to the dynamic stiffness on the component interface, and

$$
\boldsymbol{D}_{j j}^{*}(\lambda)=\left(\boldsymbol{K}_{j j}-\lambda \boldsymbol{M}_{j j}\right)-\left(\boldsymbol{K}_{j i}-\lambda \boldsymbol{M}_{j i}\right)\left(\boldsymbol{K}_{i i}-\lambda \boldsymbol{M}_{i i}\right)^{-1}\left(\boldsymbol{K}_{i j}-\lambda \boldsymbol{M}_{i j}\right)
$$

On the basis of Equation (12), Equation (15) can be expressed by making use of the main modes on the fixed interface, as

$$
\begin{aligned}
\boldsymbol{D}_{j j}^{*}(\lambda)= & \left(\boldsymbol{K}_{j j}-\lambda \boldsymbol{M}_{j j}\right)-\boldsymbol{K}_{j i} \Phi_{i i} \Delta_{i i}{ }^{-1}(\lambda) \Phi_{i i}{ }^{T} \boldsymbol{K}_{i j}+\lambda \boldsymbol{K}_{j i} \Phi_{i i} \Delta_{i i}{ }^{-1}(\lambda) \Phi_{i i}{ }^{T} \boldsymbol{M}_{i j}+ \\
& \lambda \boldsymbol{M}_{j i} \Phi_{i i} \Delta_{i i}{ }^{-1}(\lambda) \Phi_{i i}{ }^{T} \boldsymbol{K}_{i j}-\lambda^{2} \boldsymbol{M}_{j i} \Phi_{i i} \Delta_{i i}{ }^{-1}(\lambda) \Phi_{i i}{ }^{T} \boldsymbol{M}_{i j}
\end{aligned}
$$

Moreover, it is well known that

$$
\Delta_{i i}{ }^{-1}(\lambda) \Delta_{i i}(\lambda)=\boldsymbol{I}_{i i}=\Delta_{i i}{ }^{-1}(\lambda)\left(\Lambda_{i i}-\lambda \boldsymbol{I}_{i i}\right)
$$

Then, some formulations can be obtained respectively from Equation (17), as

$$
\begin{gathered}
\lambda \Delta_{i i}{ }^{-1}(\lambda)=-\boldsymbol{I}_{i i}+\Delta_{i i}{ }^{-1}(\lambda) \Lambda_{i i} \\
\lambda^{2} \Delta_{i i}{ }^{-1}(\lambda)=-\lambda \boldsymbol{I}_{i i}-\Lambda_{i i}+\Lambda_{i i} \Delta_{i i}{ }^{-1}(\lambda) \Lambda_{i i}
\end{gathered}
$$

Substituting Equation (18) and Equation (19) into Equation (16), $D_{j j}^{*}(\lambda)$ can be rewritten as follows:

$$
\begin{aligned}
\boldsymbol{D}_{j j}^{*}(\lambda)= & \boldsymbol{K}_{j j}-\boldsymbol{K}_{j i} \Phi_{i i} \Phi_{i i}{ }^{T} \boldsymbol{M}_{i j}-\boldsymbol{M}_{j i} \Phi_{i i} \Phi_{i i}{ }^{T} \boldsymbol{K}_{i j}+\boldsymbol{M}_{j i} \Phi_{i i} \Lambda_{i i} \Phi_{i i}{ }^{T} \boldsymbol{M}_{i j}- \\
& \lambda\left(\boldsymbol{M}_{j j}-\boldsymbol{M}_{j i} \Phi_{i i} \Phi_{i i}{ }^{T} \boldsymbol{M}_{i j}\right)-\left(\boldsymbol{K}_{j i} \Phi_{i i}-\boldsymbol{M}_{j i} \Phi_{i i} \Lambda_{i i}\right) \Delta_{i i}{ }^{-1}(\lambda)\left(\Phi_{i i}{ }^{T} \boldsymbol{K}_{i j}-\Lambda_{i i} \Phi_{i i}{ }^{T} \boldsymbol{M}_{i j}\right)
\end{aligned}
$$

By making use of Equation (7) and the orthogonal modes, Equation (20) can be expressed finally, as follows:

$$
\begin{aligned}
& \boldsymbol{D}_{j j}^{*}(\lambda)=\boldsymbol{K}_{j j}-\boldsymbol{K}_{j i} \Phi_{i i} \Phi_{i i}{ }^{T} \boldsymbol{M}_{i j}-\boldsymbol{M}_{j i} \Phi_{i i} \Phi_{i i}{ }^{T} \boldsymbol{K}_{i j}+ \\
& \boldsymbol{M}_{j i} \Phi_{i i} \Lambda_{i i} \Phi_{i i}{ }^{T} \boldsymbol{M}_{i j}-\lambda\left(\boldsymbol{M}_{j j}-\boldsymbol{M}_{j i} \Phi_{i i} \Phi_{i i}{ }^{T} \boldsymbol{M}_{i j}\right)- \\
& \left(\begin{array}{l}
\boldsymbol{K}_{j i}-\boldsymbol{M}_{j i} \boldsymbol{M}_{i i}{ }^{-1} \boldsymbol{K}_{i i} \\
\boldsymbol{K}_{j i}-\boldsymbol{M}_{j i} \boldsymbol{M}_{i i}{ }^{-1} \boldsymbol{K}_{i i}
\end{array}\right)\left(\begin{array}{l}
\left.\Phi_{i k} \boldsymbol{\Delta}_{k k}{ }^{-1}(\lambda) \Phi_{i k}{ }^{T}\right)\left(\boldsymbol{K}_{i j}-\boldsymbol{K}_{i i} \boldsymbol{M}_{i i}{ }^{-1} \boldsymbol{M}_{i j}\right)^{-} \\
\left.\Phi_{i h} \boldsymbol{\Delta}_{h h}{ }^{-1}(\lambda) \Phi_{i h}{ }^{T}\right)\left(\boldsymbol{K}_{i j}-\boldsymbol{K}_{i i} \boldsymbol{M}_{i i}{ }^{-1} \boldsymbol{M}_{i j}\right)
\end{array}\right.
\end{aligned}
$$

On the condition that $\lambda<\lambda_{k+1}$, the variable $\Delta_{h h}{ }^{-1}(\lambda)$ can be expanded by using the Taylor expansion, as

$$
\Delta_{h h}{ }^{-1}(\lambda)=\sum_{q=0}^{\infty} \lambda^{q} \Lambda_{h h}{ }^{-(q+1)}=\Lambda_{h h}{ }^{-1}+\lambda \Lambda_{h h^{-2}}+\sum_{q=2}^{\infty} \lambda^{q} \Lambda_{h h}{ }^{-(q+1)}, q \geq 0
$$

What is more, the natural frequencies and mode shapes of the component satisfy the following equations:

$$
\begin{gathered}
\boldsymbol{K}_{i i} \Phi_{i k}=\boldsymbol{M}_{i i} \Phi_{i k} \Lambda_{k k} \\
\boldsymbol{K}_{i i}{ }^{-1}=\Phi_{i k} \Lambda_{k k}{ }^{-1} \Phi_{i k}{ }^{T}+\Phi_{i h} \Lambda_{h h}{ }^{-1} \Phi_{i h}{ }^{T} \\
\boldsymbol{K}_{i i}{ }^{-1} \boldsymbol{M}_{i i} \boldsymbol{K}_{i i}{ }^{-1}=\Phi_{i k} \Lambda_{k k}{ }^{-2} \Phi_{i k}{ }^{T}+\Phi_{i h} \Lambda_{h h}{ }^{-2} \Phi_{i h}{ }^{T} \\
\boldsymbol{M}_{i i}{ }^{-1}\left(\boldsymbol{M}_{i i} \boldsymbol{K}_{i i}{ }^{-1}\right)^{q}=\Phi_{i k} \Lambda_{k k}{ }^{-q} \Phi_{i k}{ }^{T}+\Phi_{i h} \Lambda_{h h}{ }^{-q} \Phi_{i h}{ }^{T}
\end{gathered}
$$


Substituting Equations (22) and (23-26) into Equation (21), the equation about $D_{j j}^{*}(\lambda)$ is rewritten again, as

$$
\boldsymbol{D}_{j j}^{*}(\lambda)=\kappa_{j j}-\lambda \mu_{j j}-\lambda^{2} \mu_{j k} \Delta_{k k}(\lambda) \mu_{k j}-\sum_{m=2}^{\infty} \lambda^{q} \mu_{j j}{ }^{(q)}
$$

where,

$$
\begin{gathered}
\boldsymbol{\kappa}_{j j}=\boldsymbol{K}_{j j}-\boldsymbol{K}_{j i} \boldsymbol{K}_{i i}{ }^{-1} \boldsymbol{K}_{i j} \\
\Delta_{k k}(\lambda)=\Lambda_{k k}-\lambda \boldsymbol{I}_{k k} \\
\mu_{j k}=\left(\boldsymbol{M}_{j i}-\boldsymbol{K}_{j i} \boldsymbol{K}_{i i}{ }^{-1} \boldsymbol{M}_{i i}{ }^{-1}\right) \Phi_{i k} \\
\mu_{j j}=\boldsymbol{M}_{j j}-\boldsymbol{M}_{j i} \boldsymbol{K}_{i i}{ }^{-1} \boldsymbol{K}_{i j}+\boldsymbol{K}_{j i} \boldsymbol{K}_{i i}{ }^{-1}\left(\boldsymbol{M}_{i i} \boldsymbol{K}_{i i}{ }^{-1} \boldsymbol{K}_{i j}-\boldsymbol{M}_{i j}\right) \\
\mu_{j j}{ }^{(q)}=\left(\boldsymbol{M}_{j i}-\boldsymbol{K}_{j i} \boldsymbol{K}_{i i}{ }^{-1} \boldsymbol{M}_{i i}\right)\left(\boldsymbol{K}_{i i}{ }^{-1}-\Phi_{i k} \Lambda_{k k}{ }^{-2} \Phi_{i k}{ }^{T}\right)\left(\boldsymbol{M}_{i i} \boldsymbol{K}_{i i}{ }^{-1}\right)^{q-2}\left(\boldsymbol{M}_{i j}-\boldsymbol{M}_{i i} \boldsymbol{K}_{i i}{ }^{-1} \boldsymbol{K}_{i j}\right) .
\end{gathered}
$$

Substituting Equation (27) into Equations (13) and (14), the equation about $f_{j}(\lambda)$ can be rewritten as follows:

$$
\begin{gathered}
\boldsymbol{f}_{j}(\lambda)=-\lambda \mu_{j k} \boldsymbol{H}_{k}+\left[\kappa_{j j}-\lambda \mu_{j j}-\sum_{q=2}^{\infty} \lambda^{q} \mu_{j j}(q)\right] \boldsymbol{X}_{j} \\
\boldsymbol{H}_{k}=\lambda \Delta_{k k}{ }^{-1}(\lambda) \mu_{k j} \boldsymbol{X}_{j}
\end{gathered}
$$

Additionally, the constraint equation of the component can be achieved as below

$$
\left(\Lambda_{k k}-\lambda \boldsymbol{I}_{k k}\right) \boldsymbol{H}_{k}-\lambda \mu_{k j} \boldsymbol{X}_{j}=\mathbf{0}_{k}
$$

Subsequently, the relationships between the interface force and the displacement of the disk and hard-coated blades can be obtained from Equation (33), which are, respectively:

$$
\begin{aligned}
& f_{j}^{\mathrm{b}}(\lambda)=-\lambda \mu_{j k}^{\mathrm{b}} \boldsymbol{H}_{k}^{\mathrm{b}}+\left[\kappa_{j j}^{\mathrm{b}}-\lambda \mu_{j j}^{\mathrm{b}}-\sum_{q=2}^{\infty} \lambda^{q} \mu_{j j}^{\mathrm{b}}(q)\right] \boldsymbol{X}_{j}^{\mathrm{b}} \\
& f_{j}^{\mathrm{d}}(\lambda)=-\lambda \mu_{j k}^{\mathrm{d}} \boldsymbol{H}_{k}^{\mathrm{d}}+\left[\kappa_{j j}^{\mathrm{d}}-\lambda \mu_{j j}^{\mathrm{d}}-\sum_{q=2}^{\infty} \lambda^{q} \mu_{j j}^{\mathrm{d}}(q)\right] \boldsymbol{X}_{j}^{\mathrm{d}}
\end{aligned}
$$

For realizing the modal synthesis of the HCM blisk accurately, the double coordinating conditions of the interface displacement and force are introduced together, as below:

$$
\begin{gathered}
X_{j}^{\mathrm{b}}=X_{j}^{\mathrm{d}} \\
f_{j}^{\mathrm{b}}+f_{j}^{\mathrm{d}}=\mathbf{0}
\end{gathered}
$$

Then, the equilibrium and constraint equations of the HCM blisk are achieved, respectively, as

$$
\begin{gathered}
-\lambda \widetilde{\mu}_{j k} \widetilde{\boldsymbol{H}}_{k}+\left[\widetilde{\kappa}_{j j}-\lambda \widetilde{\mu}_{j j}-\sum_{q=2}^{\infty} \lambda{ }^{q} \widetilde{\mu}_{j j}^{(q)}\right] \widetilde{\boldsymbol{X}}_{j}=0_{j} \\
\Delta_{k k}(\lambda) \widetilde{\boldsymbol{H}}_{k}-\lambda \widetilde{\mu}_{k j} \widetilde{\boldsymbol{X}}_{j}=\mathbf{0}_{j}
\end{gathered}
$$

Further, by making use of Equations (40) and (41) together, Equation (42) can be deduced as

$$
\left\{\left[\begin{array}{cc}
\widetilde{\kappa}_{j j} & \mathbf{0}_{j k} \\
\mathbf{0}_{k j} & \widetilde{\Lambda}_{k k}
\end{array}\right]-\lambda\left[\begin{array}{cc}
\widetilde{\mu}_{i j} & \widetilde{\mu}_{j k} \\
\widetilde{\mu}_{j k}^{T} & \boldsymbol{I}_{k k}
\end{array}\right]-\sum_{q=2}^{\infty} \lambda^{q}\left[\begin{array}{cc}
\widetilde{\mu}_{j j}^{(q)} & \mathbf{0}_{j k} \\
\mathbf{0}_{k j} & \mathbf{0}_{k k}
\end{array}\right]\right\}\left\{\begin{array}{l}
\widetilde{\boldsymbol{X}}_{j} \\
\widetilde{\boldsymbol{H}}_{k}
\end{array}\right\}=\left\{\begin{array}{l}
\mathbf{0}_{j} \\
\mathbf{0}_{k}
\end{array}\right\}
$$


where,

$$
\begin{gathered}
\widetilde{\kappa}_{j j}=\kappa_{j j}^{\mathrm{b}}+\kappa_{j j}^{\mathrm{d}} \\
\widetilde{\mu}_{j j}=\mu_{j j}^{\mathrm{b}}+\mu_{j j}^{\mathrm{d}} \\
\widetilde{\mu}_{j k}=\left[\begin{array}{ll}
\mu_{j k}^{\mathrm{b}} & \mu_{j k}^{\mathrm{d}}
\end{array}\right] \\
\widetilde{\mu}_{j j}^{(q)}=\mu_{j j}^{\mathrm{b}(q)}+\mu_{j j}^{\mathrm{d}(q)}, q \geq 2 \\
\widetilde{\boldsymbol{H}}_{k}=\left[\begin{array}{ll}
\boldsymbol{H}_{k}^{\mathrm{b}} & \boldsymbol{H}_{k}^{\mathrm{d}}
\end{array}\right]^{T} \\
\widetilde{\Lambda}_{k k}=\operatorname{Diag}\left[\begin{array}{ll}
\Lambda_{k k}^{\mathrm{b}} & \Lambda_{k k}^{\mathrm{d}}
\end{array}\right]
\end{gathered}
$$

In particular, the first row of Equation (42) can be expanded and then expressed as follows:

$$
\widetilde{\kappa}_{j j}-\lambda\left(\widetilde{\mu}_{j j}-\widetilde{\mu}_{j k} \widetilde{\mu}_{j k}^{T}\right)-\sum_{q=2}^{\infty} \lambda^{q} \widetilde{\mu}_{j j}^{(q)}-\widetilde{\mu}_{j k} \widetilde{\Lambda}_{k k} \widetilde{\boldsymbol{H}}_{k}=\mathbf{0}
$$

Multiplying Equation (49) by $\lambda^{n}(1 \leq n \leq m)$, Equation (50) can be obtained based on the mathematical induction

$$
\left[\sum_{p=1}^{n+1} \lambda^{q} \widetilde{\mu}_{j k} \widetilde{\Lambda}_{k k}^{n-q+1} \widetilde{\mu}_{j k}^{T}+\lambda^{n} \widetilde{\kappa}_{j j}-\lambda^{n+1} \widetilde{\mu}_{j j}-\sum_{q=n+2}^{\infty} \lambda^{q} \widetilde{\mu}_{j j}^{(n-q)}\right] \widetilde{\boldsymbol{X}}_{j}=\widetilde{\mu}_{j k} \widetilde{\Lambda}_{k k}^{n+1} \widetilde{\boldsymbol{H}}_{k} .
$$

In Equation (50), $\lambda^{q}(q>m)$, which is related to the high derivative, is eliminated, then Equation (51) is achieved, as

$$
\widetilde{\boldsymbol{X}}_{h}=\boldsymbol{A}^{-1} \boldsymbol{B} \widetilde{\boldsymbol{H}}_{k}
$$

where,

$$
\begin{gathered}
\boldsymbol{A}=\left[\begin{array}{cccccc}
\widetilde{\kappa}_{j j}+\widetilde{\mu}_{j k} \widetilde{\Lambda}_{k k} \widetilde{\mu}_{j k}^{T} & \widetilde{\mu}_{j k} \widetilde{\mu}_{j k}^{T}-\widetilde{\mu}_{j j} & -\widetilde{\mu}_{j j}^{(2)} & \cdots & -\widetilde{\mu}_{j j}^{(m-1)} \\
\widetilde{\mu}_{j k} \widetilde{\Lambda}_{k k}^{2} \widetilde{\mu}_{j k}^{T} & \widetilde{\boldsymbol{\kappa}}_{j j}+\widetilde{\mu}_{j k} \widetilde{\Lambda}_{k k} \widetilde{\mu}_{j k}^{T} & \widetilde{\mu}_{j k} \widetilde{\mu}_{j k}^{T}-\widetilde{\mu}_{j j} & \cdots & -\widetilde{\mu}_{j j}^{(m-2)} \\
\widetilde{\mu}_{j k} \widetilde{\Lambda}_{k k}^{3} \widetilde{\mu}_{j k}^{T} & \widetilde{\mu}_{j k} \widetilde{\Lambda}_{k k}^{2} \widetilde{\mu}_{j k}^{T} & \widetilde{\kappa}_{j j}+\widetilde{\mu}_{j k} \widetilde{\Lambda}_{k k} \widetilde{\mu}_{j k}^{T} & \cdots & -\widetilde{\mu}_{j j}^{(m-3)} \\
\vdots & \vdots & \vdots & \ddots & \vdots \\
\widetilde{\mu}_{j k} \widetilde{\Lambda}_{k k}^{m} \widetilde{\mu}_{j k}^{T} & \widetilde{\mu}_{j k} \widetilde{\Lambda}_{k k}^{(m-1)} \widetilde{\mu}_{j k}^{T} & \widetilde{\mu}_{j k} \widetilde{\Lambda}_{k k}^{(m-2)} \widetilde{\mu}_{j k}^{T} & \cdots & \widetilde{\kappa}_{j j}+\widetilde{\mu}_{j k} \widetilde{\Lambda}_{k k} \widetilde{\mu}_{j k}^{T}
\end{array}\right] \\
\boldsymbol{B}=\left[\begin{array}{lllllll}
\widetilde{\mu}_{j k} \widetilde{\Lambda}_{k k}^{2} & \widetilde{\mu}_{j k} \widetilde{\Lambda}_{k k}^{3} & \widetilde{\mu}_{j k} \widetilde{\Lambda}_{k k}^{4} & \cdots & \widetilde{\mu}_{j k} \widetilde{\Lambda}_{k k}^{m+1}
\end{array}\right]^{T} \\
\widetilde{\boldsymbol{X}}_{h}=\left[\begin{array}{ccccccc}
\widetilde{\boldsymbol{X}}_{h 1} & \widetilde{\boldsymbol{X}}_{h 2} & \widetilde{\boldsymbol{X}}_{h 3} & \cdots & \widetilde{\boldsymbol{X}}_{h m}
\end{array}\right]^{T}=\lambda\left[\begin{array}{lllll}
\widetilde{\boldsymbol{X}}_{j} & \widetilde{\boldsymbol{X}}_{h 1} & \widetilde{\boldsymbol{X}}_{h 2} & \cdots & \widetilde{\boldsymbol{X}}_{h(m-1)}
\end{array}\right]^{T}
\end{gathered}
$$

By making full use of Equation (51), Equation (49) can be derived again and then rewritten as follows:

$$
\begin{gathered}
\left(\widetilde{\kappa}_{j j}-\lambda \widetilde{\mu}_{j j}\right) \widetilde{\boldsymbol{X}}_{j}-\lambda\left(\widetilde{\mu}_{j k} \widetilde{\boldsymbol{H}}_{k}+\widetilde{\mu}_{j h} \widetilde{\boldsymbol{H}}_{h}\right)=\mathbf{0}_{j} \\
\widetilde{\mu}_{j h}=\left[\begin{array}{lllll}
\widetilde{\boldsymbol{\mu}}_{j j}^{(2)} & \widetilde{\mu}_{j j}^{(3)} & \ldots & \widetilde{\mu}_{j j}^{(m)} & \mathbf{0}_{j j}
\end{array}\right]
\end{gathered}
$$

By taking advantage of Equations (41), (54), and (55), (56) Equation (57) can be computed as follows:

$$
\left\{\left[\begin{array}{ccc}
\widetilde{\kappa}_{j j} & \mathbf{0}_{j k} & \mathbf{0}_{j h} \\
\mathbf{0}_{k j} & \widetilde{\Lambda}_{k k} & \mathbf{0}_{h k} \\
\mathbf{0}_{h j} & \mathbf{0}_{k h} & \widetilde{\kappa}_{h h}
\end{array}\right]-\lambda\left[\begin{array}{ccc}
\widetilde{\mu}_{i j} & \widetilde{\mu}_{j k} & \widetilde{\mu}_{j h} \\
\widetilde{\mu}_{j k}^{T} & \mathbf{I}_{k k} & \mathbf{0}_{k h} \\
\widetilde{\mu}_{j h}^{T} & \mathbf{0}_{h k} & \widetilde{\mu}_{h h}
\end{array}\right]\right\}\left\{\begin{array}{c}
\widetilde{\boldsymbol{X}}_{j} \\
\widetilde{\boldsymbol{H}}_{k} \\
\widetilde{\boldsymbol{X}}_{h}
\end{array}\right\}=\left\{\begin{array}{c}
\mathbf{0}_{j} \\
\mathbf{0}_{k} \\
\mathbf{0}_{h}
\end{array}\right\}
$$


where,

$$
\begin{gathered}
\widetilde{\kappa}_{h h}=\left[\begin{array}{ccccc}
\widetilde{\mu}_{j j}^{(2)} & \widetilde{\mu}_{j j}^{(3)} & \ldots & \widetilde{\mu}_{j j}^{(m)} & \mathbf{0}_{j j} \\
\widetilde{\mu}_{j j}^{(3)} & \widetilde{\mu}_{j j}^{(4)} & \ldots & \mathbf{0}_{j j} & \mathbf{0}_{j j} \\
\vdots & \vdots & . \cdot & \vdots & \vdots \\
\widetilde{\mu}_{j j}^{(m)} & \mathbf{0}_{j j} & \cdots & \mathbf{0}_{j j} & \mathbf{0}_{j j} \\
\mathbf{0}_{j j} & \mathbf{0}_{j j} & \cdots & \mathbf{0}_{j j} & \mathbf{0}_{j j}
\end{array}\right]_{m \times m} \\
\widetilde{\mu}_{h h}=\left[\begin{array}{cccccc}
\widetilde{\mu}_{j j}^{(3)} & \widetilde{\mu}_{j j}^{(4)} & \ldots & \widetilde{\mu}_{j j}^{(m)} & \mathbf{0}_{j j} & \mathbf{0}_{j j} \\
\widetilde{\mu}_{j j}^{(4)} & . \cdot & . & . \cdot & . & \mathbf{0}_{j j} \\
\vdots & . \cdot & . & . \cdot & . & \vdots \\
\widetilde{\mu}_{j j}^{(m)} & . \cdot & . & . \cdot & . \cdot & \mathbf{0}_{j j} \\
\mathbf{0}_{j j} & . \cdot & . \cdot & . \cdot & . \cdot & \mathbf{0}_{j j} \\
\mathbf{0}_{j j} & \mathbf{0}_{j j} & \cdots & \mathbf{0}_{j j} & \mathbf{0}_{j j} & \mathbf{0}_{j j}
\end{array}\right]_{m \times m}
\end{gathered}
$$

Moreover, Equation (60) can be obtained from the expansion of Equation (51), and given by

$$
\left\{\begin{array}{c}
\widetilde{\boldsymbol{X}}_{j} \\
\widetilde{\boldsymbol{H}}_{k} \\
\widetilde{\boldsymbol{X}}_{h}
\end{array}\right\}=\left[\begin{array}{cc}
\boldsymbol{I}_{j j} & \mathbf{0}_{j k} \\
\mathbf{0}_{k j} & \boldsymbol{I}_{k k} \\
\mathbf{0}_{h j} & \boldsymbol{A}^{-1} \boldsymbol{B}
\end{array}\right]\left\{\begin{array}{c}
\widetilde{\boldsymbol{X}}_{j} \\
\widetilde{\boldsymbol{H}}_{k}
\end{array}\right\}=\boldsymbol{T}\left\{\begin{array}{c}
\widetilde{\boldsymbol{X}}_{j} \\
\widetilde{\boldsymbol{H}}_{k}
\end{array}\right\}
$$

Substituting Equation (60) into Equation (57) and pre-multiplying that by $\boldsymbol{T}^{T}$, Equation (60) can be achieved, as

$$
(\kappa-\lambda \mu)\left\{\begin{array}{c}
\widetilde{\boldsymbol{X}}_{j} \\
\widetilde{\boldsymbol{H}}_{k}
\end{array}\right\}=\left\{\begin{array}{c}
\mathbf{0}_{j} \\
\mathbf{0}_{k}
\end{array}\right\}
$$

Where $\kappa$ and $\mu$ are the reduced stiffness and mass matrices of the HCM blisk, respectively, and

$$
\begin{gathered}
\kappa=\left[\begin{array}{cc}
\widetilde{\kappa}_{j j} & \mathbf{0}_{j k} \\
\mathbf{0}_{k j} & \widetilde{\Lambda}_{k k}+\left(\boldsymbol{A}^{-1} \boldsymbol{B}\right)^{T} \widetilde{\kappa}_{h h} \boldsymbol{A}^{-1} \boldsymbol{B}
\end{array}\right] \\
\boldsymbol{\mu}=\left[\begin{array}{cc}
\widetilde{\mu}_{j j} & \widetilde{\mu}_{j k}+\widetilde{\mu}_{j h} \boldsymbol{A}^{-1} \boldsymbol{B} \\
\left(\widetilde{\mu}_{j k}+\widetilde{\mu}_{j h} \boldsymbol{A}^{-1} \boldsymbol{B}\right)^{T} & \boldsymbol{I}_{k k}+\left(\boldsymbol{A}^{-1} \boldsymbol{B}\right)^{T} \widetilde{\mu}_{h h} \boldsymbol{A}^{-1} \boldsymbol{B}
\end{array}\right]
\end{gathered}
$$

\subsection{Solution for the Vibration Characteristics of the HCM Blisk}

The natural frequencies and mode shapes of the HCM blisk, $\omega_{\mathrm{r}}$ and $\varphi_{\mathrm{r}}$, can be calculated by making use of the reduced stiffness and mass matrices to obtain the eigenvalue equation, that is

$$
\left(\kappa-\omega^{2} \mu\right) \varphi=0
$$

In general, the modal loss factors are the key characteristics of the vibrating structure, which can exhibit a damping capacity. For composite structures, an energy-based method is currently becoming more widely used by researchers for the purpose of estimating the modal loss factors of interest; examples are Johnson and Kienholz in 1982 [26], Zheng et al. in 2006 [27], Sinha in 2009 [28], and Curà et al. in 2012 [29]. In this paper, the modal loss factor of the HCM blisk, $\eta_{r}$, can be achieved by taking advantage of the modal strain energy method (MSEM), that is given as follows:

$$
\eta_{\mathrm{r}}=\frac{\eta_{\mathrm{c}} \times \mathrm{MSE}_{\mathrm{hc}, \mathrm{r}}+\eta_{\mathrm{b}} \times \mathrm{MSE}_{\mathrm{bd}, \mathrm{r}}}{\mathrm{MSE}_{\mathrm{hc}, \mathrm{r}}+\mathrm{MSE}_{\mathrm{bd}, \mathrm{r}}}
$$


What is more, because the value of $\eta_{c}$ is far less than the value of $\eta_{b}, \eta_{r}$ can also be expressed as

$$
\eta_{\mathrm{r}}=\frac{\mathrm{MSE}_{\mathrm{bd}, \mathrm{r}}}{\mathrm{MSE}_{\mathrm{hc}, \mathrm{r}}+\mathrm{MSE}_{\mathrm{bd}, \mathrm{r}}} \times \eta_{\mathrm{b}}
$$

where $\eta_{\mathrm{b}}$ and $\eta_{\mathrm{c}}$ refer to the material loss factor of the mistuned blisk and hard coating, respectively, and $\mathrm{MSE}_{\mathrm{bd}, \mathrm{r}}$ and $\mathrm{MSE}_{\mathrm{hc}, \mathrm{r}}$ refer to the modal strain energy of the mistuned blisk and hard coating, respectively.

As is known, it is very difficult to calculate and measure the damping matrix of a vibrating structure, especially for the HCM blisk, which is a treated as a complex and composite structure generally. Because of the nicer stability of hard coating under severe conditions, the HCM blisk can be thought of as a special homologous liner system. Thus, the proportional damping model [30], which features a concise implementation and simplified calculations, can reasonably be applied to the HCM blisk for the purpose of capturing its damping behavior. Supposing $\alpha$ and $\beta$ as the proportional constants, the damping matrix of the HCM blisk, $D$, can be achieved as

$$
\begin{gathered}
D=\alpha \mu+\beta \kappa \\
\alpha+\beta \omega_{\mathrm{r}}^{2}=\eta_{\mathrm{r}} \omega_{\mathrm{r}}
\end{gathered}
$$

Finally, the frequency response function of the HCM blisk, $H(\omega)$, which demonstrates the dynamic behavior of the HCM blisk, can be computed as follows:

$$
H(\omega)=\sum_{\mathrm{r}=1}^{m} \frac{\varphi_{\mathrm{r}} \varphi_{\mathrm{r}}^{T}}{\omega_{\mathrm{r}}^{2}-\omega^{2}+\mathrm{i} \omega D_{\mathrm{r}}}
$$

where $\varphi_{\mathrm{r}}$ is the regularized mode vectors of the $r$-th mode.

\section{Numerical Results of the Case Study}

\subsection{Description of the HCM Blisk and Experimental Test}

A mistuned blisk made of aluminum and devices including an exciting hammer, a specific fixture, a B\&K-4517 acceleration transducer, a LMS16 front-end data acquisition controller (FEDAC, LMS, Rouwen, Belgium), and a mobile workstation that had installed on it the LMS Test. lab, is shown in Figure 4. A NiCoCrAlY + YSZ hard coating is deposited on both sides of all of the blades of the mistuned blisk by atmospheric plasma spraying (APS) technology with coating thickness $h=0.2 \mathrm{~mm}$, as shown in Figure 5.

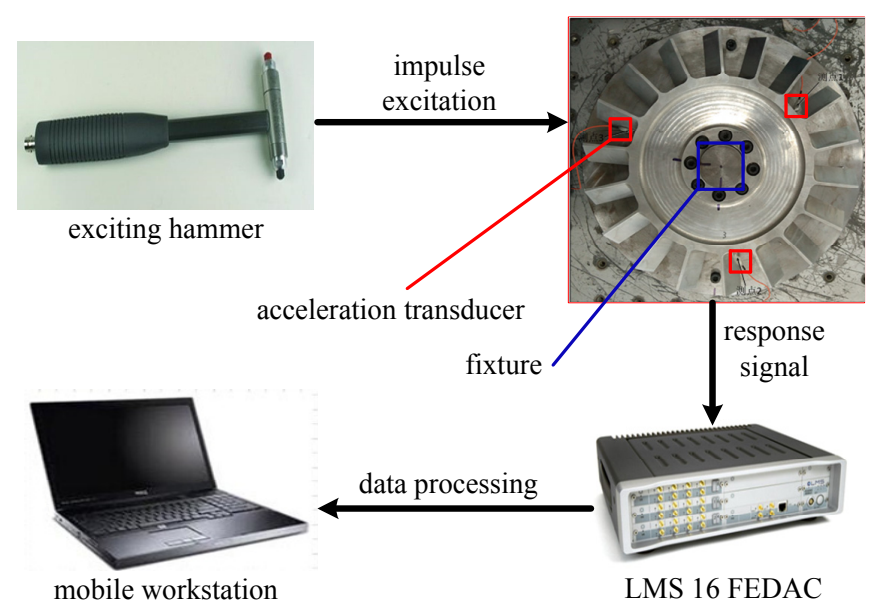

Figure 4. Picture of the mistuned blisk and experimental devices. FEDAC, front-end data acquisition controller. 



Figure 5. Mistuned blisk with a hard coating of NiCoCrAlY + YSZ.

The nominal geometric and mechanical parameters associated with the mistuned blisk and the NiCoCrAlY + YSZ hard coating are given in Tables 1 and 2, respectively. The Young's modulus and the material loss factor of the NiCoCrAlY + YSZ hard coating are obtained from a dynamic thermo-mechanical analysis (DMA) and the inverse method at room temperature, respectively [31].

Table 1. Geometrical parameters of the mistuned blisk and hard coating.

\begin{tabular}{cccc}
\hline \multicolumn{1}{c}{ Disk } & & \multicolumn{2}{c}{ Hard-Coated Blades } \\
\hline Inner radius of hub & $50 \mathrm{~mm}$ & Height of blade & $50 \mathrm{~mm}$ \\
Outer radius of hub & $80 \mathrm{~mm}$ & Width of blade & $25 \mathrm{~mm}$ \\
Thickness of hub & $19 \mathrm{~mm}$ & Thickness of blade & $3 \mathrm{~mm}$ \\
Inner radius of rim & $182 \mathrm{~mm}$ & Number of blade & 18 \\
Outer radius of rim & $200 \mathrm{~mm}$ & Coating thickness & $0.2 \mathrm{~mm}$ \\
Thickness of rim & $20 \mathrm{~mm}$ & Coating area & $100 \%$ \\
Thickness of sternum & $7 \mathrm{~mm}$ & Coating location & Both sides \\
\hline
\end{tabular}

Table 2. Mechanical parameters of the mistuned blisk and hard coating.

\begin{tabular}{ccc}
\hline Type & Blisk & Hard Coating \\
\hline Material & Aluminum & NiCoCrAlY + YSZ \\
Young's modulus $(\mathrm{Gpa})$ & 70 & 54.494 \\
Mass density $\left(\mathrm{Kg} / \mathrm{m}^{3}\right)$ & 2700 & 5600 \\
Loss factor & 0.0003 & 0.0212 \\
Poisson's ratio & 0.33 & 0.3 \\
\hline
\end{tabular}

\subsection{Vibration Characteristics of the HCM Blisk and Verification}

On basis of the proposed ROM, the vibration characteristics of the HCM blisk, including the natural frequencies, mode shapes, modal loss factors and the frequency response function, are solved. For the purpose of verifiying the validation of the proposed ROM, the results are compared with those obtained from the full-order model (FOM) of the HCM blisk established in the commercial software ANSYS 15.0 Rel. (ANSYS, San Francisco, CA, USA), which possesses approximately 4.23 million SOLID185 elements and 13.85 million DOFs.

Moreover, in contrast with a FEA of the HCM blisk, the hammering method can be employed in an experimental test to obtain the natural frequencies, mode shapes and modal loss factors (modal damping ratio). In accordance with the peening point marked in the Geometry module of LMS Test.lab (LMS, Rouwen, Belgium), the HCM blisk fixed on the specific fixture is peened by the exciting hammer. By using three lightweight acceleration transducer B\&K-4517s equispaced on the root of the 
hard-coated blade, the response single can be transmitted to the LMS16 FEDAC, which is responsible for the data's acquisition and analysis. The natural frequencies, modal loss factors, and the mode shapes of the HCM blisk are extracted from the PolyMAX module of LMS Test.Lab.

The natural frequencies obtained from the ROM, the FOM, and the experimental test are listed in Table 3. It can be seen that the results have similar consistency in general, i.e., $600-800 \mathrm{~Hz}$ and 1100-1400 Hz. Moreover, although the natural frequencies obtained from the ROM are different from the others, the error between the ROM and the FOM, ER-F, or the test, ER-T, is not large; in fact, they are $0.47 \%-1.89 \%$ and $2.40 \%-4.89 \%$, respectively.

Table 3. Natural frequencies obtained from the ROM, full-order model (FOM), and the test (Hz).

\begin{tabular}{cccccc}
\hline Mode Order & ROM & FOM & Test & $E_{\boldsymbol{R}-\boldsymbol{F}}$ & $\boldsymbol{E}_{\boldsymbol{R}-\boldsymbol{T}}$ \\
\hline 1 & 646.20 & 651.12 & 662.09 & $0.76 \%$ & $2.40 \%$ \\
2 & 654.62 & 663.21 & 672.68 & $1.29 \%$ & $2.68 \%$ \\
3 & 667.92 & 674.75 & 688.59 & $1.01 \%$ & $3.01 \%$ \\
4 & 677.77 & 687.54 & 712.07 & $1.42 \%$ & $4.82 \%$ \\
5 & 709.28 & 700.88 & 735.37 & $-1.20 \%$ & $3.55 \%$ \\
6 & 714.60 & 709.18 & 750.72 & $-0.76 \%$ & $4.81 \%$ \\
7 & 724.93 & 717.14 & 753.22 & $-1.09 \%$ & $3.76 \%$ \\
8 & 728.88 & 725.47 & 763.48 & $-0.47 \%$ & $4.53 \%$ \\
9 & 730.90 & 739.42 & 766.64 & $1.15 \%$ & $4.66 \%$ \\
10 & 731.65 & 745.49 & 769.29 & $1.86 \%$ & $4.89 \%$ \\
11 & 1149.10 & 1169.57 & 1193.26 & $1.75 \%$ & $3.70 \%$ \\
12 & 1237.70 & 1251.40 & 1286.64 & $1.10 \%$ & $3.80 \%$ \\
13 & 1332.60 & 1357.76 & 1394.69 & $1.85 \%$ & $4.45 \%$ \\
\hline
\end{tabular}

The model assurance criterion (MAC) [32] has been used to conduct a quantitative assessment of the mode shapes. In this paper, the MAC of the mode shapes between the ROM and the FOM are plotted in Figure 6. The crimson quadrates located on the counter-diagonal represent that the values of the MAC are close to 1 approximately, and the rest that are painted by the white color indicate generally that the values of the MAC are very small, even nearly equal to 0 . It can be shown clearly that the mode shapes obtained from the ROM and the FOM have a good correlation.

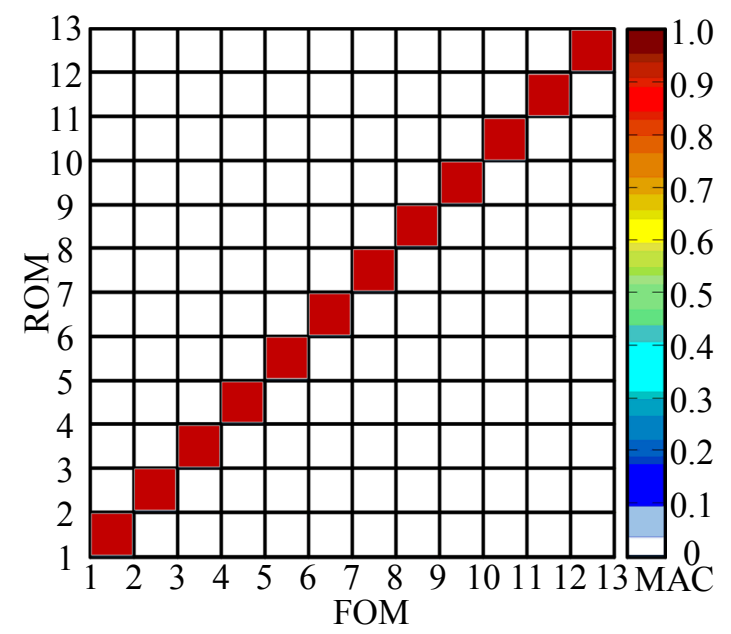

Figure 6. The model assurance criterion (MAC) of the mode shapes obtained from the ROM and the FOM.

What is more, the mode shapes when nodal diameter number $n d=1$ and 2, obtained from the ROM and experimental test, are shown in Figure 7. It can be found that the strong-coupled vibrations dominated the mode types in both results. Moreover, the blue zones represent the location at which the 
amplitudes are nearly zero, the yellow and red zones represent the location at which the amplitudes are very large, and the green zones represent the location at which the vibration amplitudes fall in between. It reveals clearly that the color distribution in the ROM and the test model is approximately similar.
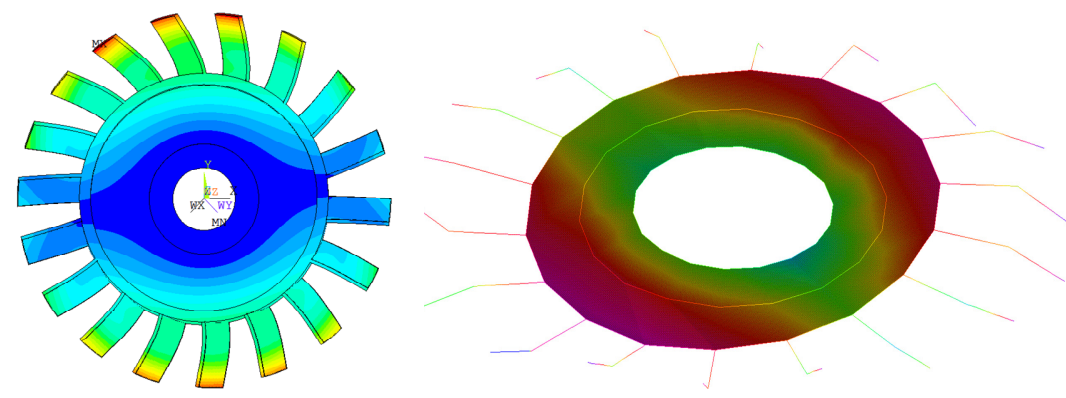

(a)
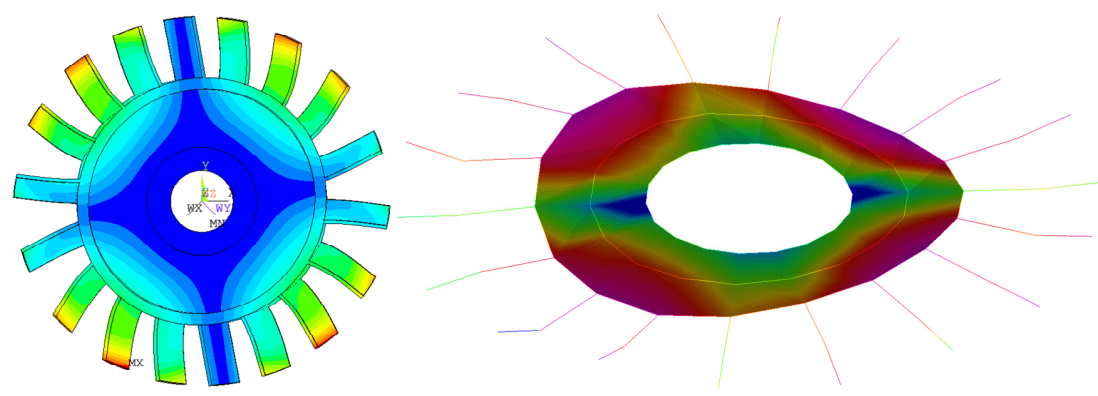

(b)

Figure 7. The comparison of mode shapes obtained from the ROM and the experimental test. (a) Mode shapes when $n d=1 ;(\mathbf{b})$ Mode shapes when $n d=2$.

\section{The Discussion of the Specific Influence of Hard Coating}

\subsection{The Influence of Hard Coating on Vibration Characteristics}

The natural frequencies of the mistuned blisk or HCM blisk obtained from the ROM and the experimental test are listed in Table 4 . The differences are calculated to investigate the specific influence of hard coating on natural frequencies. It can be found that both of them decrease similarly with the deposition of hard coating; however, the change rates of the natural frequencies are not evident. In fact, the natural frequencies obtained from the proposed ROM vary within $2.97 \%$, and those obtained from the experimental test range from $3.54 \%$ to $5.89 \%$. This means that the NiCoCrAlY + YSZ hard coating has a slight effect on natural frequencies.

Table 4. Natural frequencies of mistuned blisk and HCM blisk (Hz).

\begin{tabular}{|c|c|c|c|c|c|c|}
\hline \multirow{2}{*}{ Mode Order } & \multicolumn{3}{|c|}{ ROM } & \multicolumn{3}{|c|}{ Test } \\
\hline & Mistuned Blisk & HCM Blisk & Change Rate & Mistuned Blisk & HCM Blisk & Change Rate \\
\hline 1 & 655.99 & 646.20 & $-1.52 \%$ & 701.07 & 662.09 & $-5.89 \%$ \\
\hline 2 & 668.38 & 654.62 & $-2.10 \%$ & 710.80 & 672.68 & $-5.67 \%$ \\
\hline 3 & 676.34 & 667.92 & $-1.26 \%$ & 721.54 & 688.59 & $-4.79 \%$ \\
\hline 4 & 690.33 & 677.77 & $-1.85 \%$ & 737.30 & 712.07 & $-3.54 \%$ \\
\hline 5 & 716.70 & 709.28 & $-1.05 \%$ & 764.01 & 735.37 & $-3.89 \%$ \\
\hline 6 & 725.95 & 714.60 & $-1.59 \%$ & 780.04 & 750.72 & $-3.91 \%$ \\
\hline 7 & 733.27 & 724.93 & $-1.15 \%$ & 783.49 & 753.22 & $-4.02 \%$ \\
\hline 8 & 741.11 & 728.88 & $-1.68 \%$ & 794.87 & 763.48 & $-4.11 \%$ \\
\hline 9 & 743.69 & 730.90 & $-1.75 \%$ & 798.59 & 766.64 & $-4.17 \%$ \\
\hline 10 & 745.74 & 731.65 & $-1.93 \%$ & 801.76 & 769.29 & $-4.22 \%$ \\
\hline 11 & 1162.90 & 1149.10 & $-1.20 \%$ & 1242.86 & 1193.26 & $-4.16 \%$ \\
\hline 12 & 1272.40 & 1237.70 & $-2.80 \%$ & 1352.50 & 1286.64 & $-5.12 \%$ \\
\hline 13 & 1372.20 & 1332.60 & $-2.97 \%$ & 1469.73 & 1394.69 & $-5.38 \%$ \\
\hline
\end{tabular}


Moreover, the modal loss factors of the mistuned blisk and HCM blisk obtained from the ROM and the experimental test are shown in Figure 8. Due to the restrictions of existing damping theory for vibrating structures and the external disturbance of the test conditions, modal loss factors are generally captured within an inevitable tolerance, thus the modal loss factors obtained from the ROM and the experimental test are different. However, the general variation trends are very similar. In addition, unlike the natural frequencies, the modal loss factors do increase remarkably-more than quadruple-which means a favorable damping effect of the NiCoCrAlY + YSZ hard coating on the mistuned blisk.



Figure 8. Modal loss factors of the mistuned blisk and HCM blisk.

The frequency response functions of the mistuned blisk and the HCM blisk, ranging from 600 to $800 \mathrm{~Hz}$ (closely spaced modes), are shown in Figure 9. It can be found that the amplitudes of the mistuned blisk apparently declined without a significant variation of response frequency, which indicates that the NiCoCrAlY + YSZ hard coating does have an obvious vibration reduction on the mistuned blisk. It is noteworthy that the decrease of amplitudes corresponds to the increase of modal loss factors caused by the NiCoCrAlY + YSZ hard coating.

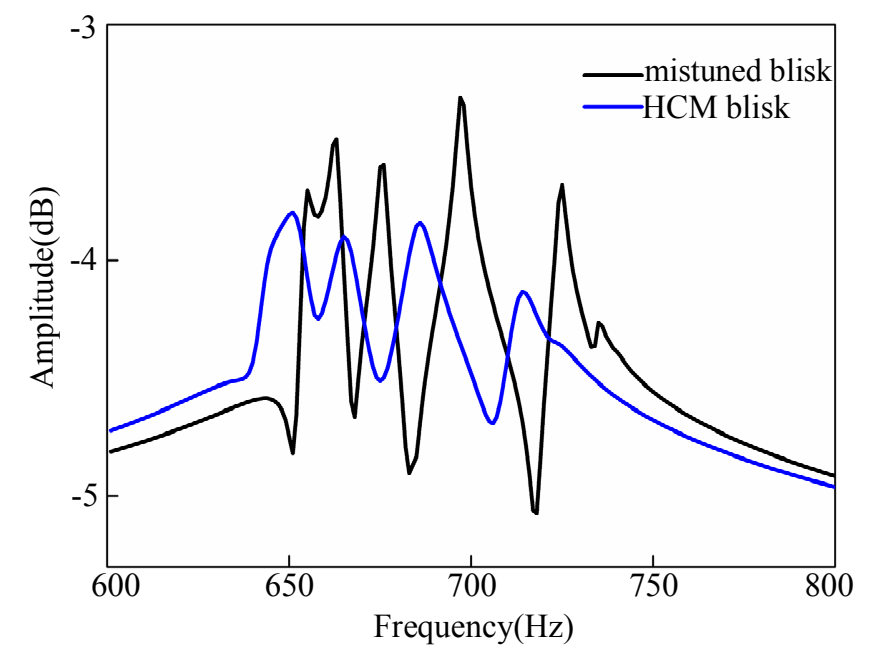

Figure 9. Frequency response functions of mistuned blisk and HCM blisk. 


\subsection{The Influence of Coating Area on Damping Capacity}

Variations of coating area are most likely to be realized in industrial applications, thus it is very significant to investigate the influence of coating area on the vibration characteristics of the mistuned blisk. Since damping capacity always plays a key role in the vibration reduction of the mistuned blisk, it should be studied according to both modal loss factors and the frequency response function in this paper. Mistuned blisks with different coating areas, classified as $0 \%, 25 \%, 50 \%, 75 \%$, and $100 \%$, are shown in Figure 10. Specific blisk areas-a portion of the blade and the whole disk-are protected by the high temperature masking tape, while the rest has a deposit of NiCoCrAlY + YSZ hard coating [33].



(a) $0 \%$



(b) $25 \%$



(c) $50 \%$



(d) $75 \%$

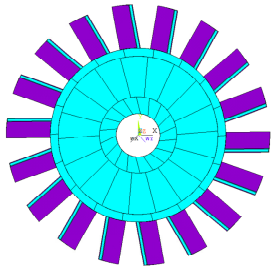

(e) $100 \%$

Figure 10. Mistuned blisks with different coating areas. (a) $0 \%$; (b) $25 \%$; (c) $50 \%$; (d) $75 \%$ and (e) $100 \%$.

The modal loss factors of the mistuned blisks with different coating areas are plotted in Figure 11. The results reveal that the modal loss factors of a mistuned blisk become larger with the increase of coating area generally. However, the increased variations of modal loss factors decreased gradually. When the coating area $A>75 \%$, the increased variations of modal loss factors are small, and the modal loss factors of the mistuned blisk are not more particularly obvious. Therefore, a conclusion can be made that the influence of the coating area on the damping capacity of a mistuned blisk is regular, and a NiCoCrAlY + YSZ hard coating should be deposited from the blade tip to the blade root.

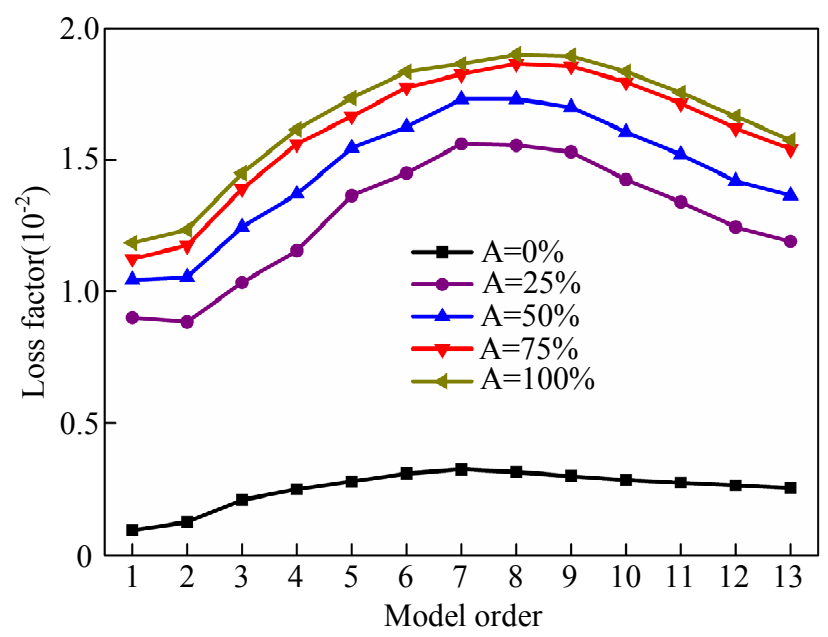

Figure 11. Modal loss factors of mistuned blisks with different coating areas.

The frequency response functions of the mistuned blisks with different coating areas are shown in Figure 12. It seems that the amplitudes of the mistuned blisks are suppressed generally at the resonant frequency, which means the damping capacity of the mistuned blisks has increased. However, the decreased gradient of the amplitudes become gradually become small. Moreover, it is worthy to note that the variation trend of the amplitudes is similar to that of the modal loss factors. 


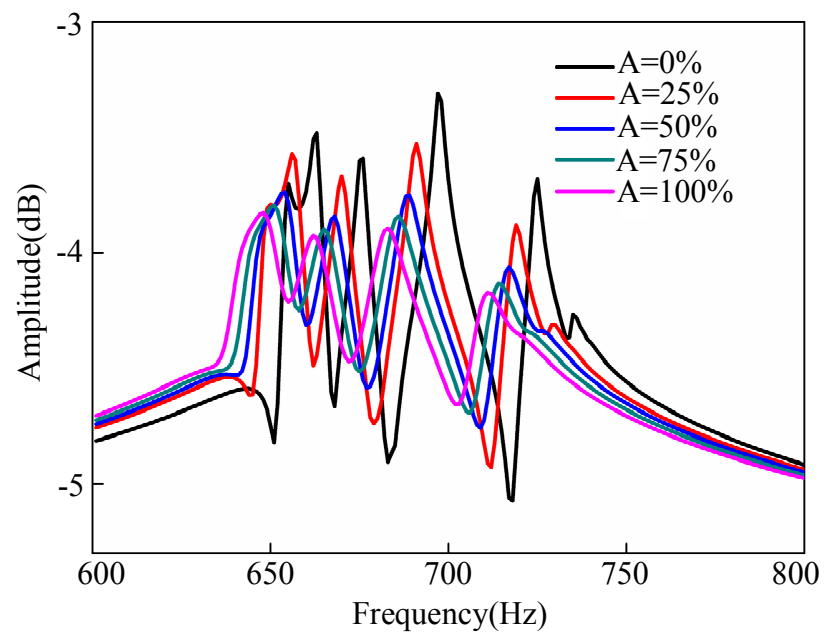

Figure 12. Frequency responses functions of mistuned blisks with different coating areas.

\section{Conclusions}

A damping strategy for the vibration reduction of a mistuned blisk by depositing hard coating on its blades was proposed and investigated in this paper. Firstly, by using an improved fixed-ICMSM, an effective ROM of a HCM blisk was established to decrease the amount of calculation while ensuring computational accuracy. Then, the vibration characteristics of the HCM blisk were obtained from the established ROM, such as the natural frequencies, mode shapes, modal loss factors and the frequency response function. Furthermore, a mistuned blisk with a deposit of NiCoCrAlY + YSZ hard coating on both sides of its blades was selected to be analyzed, and the results were compared with those obtained from the FOM and the experimental test for verifying the validation of the proposed ROM.

Moreover, the influence of the NiCoCrAlY + YSZ hard coating on the mistuned blisk was investigated in terms of natural frequencies, modal loss factors, and the frequency response function. The results reveal that the NiCoCrAlY + YSZ hard coating can bring about an apparent damping effect on the mistuned blisk with a slight variation of natural frequencies, and the resonant response of mistuned blisk in the resonant areas can be suppressed remarkably.

Finally, the influence of coating area on the damping capacity of the mistuned blisk was studied with emphasis on modal loss factors and frequency response functions. It seems that the damping capacity increases generally with the increase of coating area, but the increased gradient decreases gradually. Specifically, the variation trend of the amplitudes are similar to that of the modal loss factors.

Acknowledgments: This project is supported by the National Natural Science Foundation of China (Grant No. 51375079) and the Fundamental Research Funds for the Central Universities of China (Grant No. N150304008).

Author Contributions: Feng Gao conceived, designed and performed the experiments, analyzed the data and wrote the paper; Wei sun contributed materials and analysis tools.

Conflicts of Interest: The authors declare no conflict of interest.

\section{References}

1. Laxalde, D.; Thouverez, F.; Lombard, J.P. Forced response analysis of integrally bladed disks with friction ring dampers. J. Vib. Acoust. 2010, 132, 011013. [CrossRef]

2. Chang, Y.Y.; Lai, H.M. Wear behavior and cutting performance of CrAlSiN and TiAlSiN hard coatings on cemented carbide cutting tools for Ti alloys. Surf. Coat. Technol. 2014, 259, 152-158. [CrossRef]

3. Songbo, W.E.I.; Xiaohan, P.E.I.; Bairu, S.H.I.; Tianmin, S.H.A.O.; Tao, L.I.; Yiliang, L.I.; Yi, X.I.E. Wear resistance and anti-friction of expansion cone with hard coating. Pet. Explor. Dev. 2016, 43, 326-331. 
4. Shubin, A.Y.; Potekaev, A.I.; Savostikov, V.M.; Galsanov, S.V.; Dmitriev, V.S.; Stepanov, I.B.; Dammer, V.H. Comparative physical-tribological properties of anti-friction ion-plasma Ti-C-Mo-S coating on VT6 alloy or 20X13 and 40X steels//IOP Conference Series: Materials Science and Engineering. IOP Publ. 2017, 168,012038 .

5. Ghidelli, M.; Sebastiani, M.; Collet, C.; Guillemet, R. Determination of the elastic moduli and residual stresses of freestanding Au-TiW bilayer thin films by nanoindentation. Mater. Des. 2016, 106, 436-445. [CrossRef]

6. Doleker, K.M.; Karaoglanli, A.C. Comparison of oxidation behavior of $\mathrm{YSZ}$ and $\mathrm{Gd}_{2} \mathrm{Zr}_{2} \mathrm{O}_{7}$ thermal barrier coatings (TBCs). Surf. Coat. Technol. 2017, 318, 198-207. [CrossRef]

7. Fayomi, O.S.S.; Popoola, A.P.P.; Aigbodion, V.S. Investigation on microstructural, anti-corrosion and mechanical properties of doped $\mathrm{Zn}-\mathrm{Al}-\mathrm{SnO}_{2}$ metal matrix composite coating on mild steel. J. Alloy. Compd. 2015, 623, 328-334. [CrossRef]

8. Bakhsheshi-Rad, H.R.; Hamzah, E.; Ismail, A.F.; Daroonparvar, M.; Yajid, M.A.M. Preparation and characterization of NiCrAlY/nano-YSZ/PCL composite coatings obtained by combination of atmospheric plasma spraying and dip coating on Mg-Ca alloy. J. Alloys Compd. 2016, 658, 440-452.

9. Torvik, P.J. A slip damping model for plasma sprayed ceramics. J. Appl. Mech. 2009, 76, 061018. [CrossRef]

10. Al-Rub, R.K.K.; Palazotto, A.N. Micromechanical theoretical and computational modeling of energy dissipation due to nonlinear vibration of hard ceramic coatings with microstructural recursive faults. Int. J. Solids Struct. 2010, 47, 2131-2142. [CrossRef]

11. Torvik, P.J.; Langley, B. Material properties of hard coatings developed for high damping. In Proceedings of the 51st AIAA/SAE/ASEE Joint Propulsion Conference, Orlando, FL, USA, 27-29 July 2015.

12. Yang, Z.X.; Han, Q.K.; Jin, Z.H.; Qu, T. Solution of natural characteristics of a hard-coating plate based on Lindstedt-Poincaré perturbation method and its valedictions by FEM and measurement. Nonlinear Dyn. 2015, 81, 1207-1218. [CrossRef]

13. Sun, W.; Liu, Y.; Du, G. Analytical modeling of hard-coating cantilever composite plate considering the material nonlinearity of hard coating. Math. Probl. Eng. 2015, 2015, 978392. [CrossRef]

14. Sun, W.; Zhu, M.; Wang, Z. Free vibration analysis of a hard-coating cantilever cylindrical shell with elastic constraints. Aerosp. Sci. Technol. 2017, 63, 232-244. [CrossRef]

15. Sun, W.; Liu, Y. Vibration analysis of hard-coated composite beam considering the strain dependent characteristic of coating material. Acta Mech. Sin. 2016, 32, 731-742. [CrossRef]

16. Sun, J.; Kari, L. Coating methods to increase material damping of compressor blades: Measurements and modeling. In Proceedings of the ASME Turbo Expo 2010: Power for Land, Sea, and Air, Glasgow, UK, 14-18 June 2010; American Society of Mechanical Engineers: New York, NY, USA, 2010; pp. 1157-1165.

17. Yang, M.T.; Griffin, J.H. A reduced order model of mistuning using a subset of nominal system modes. J. Eng. Gas Turbines Power 1999, 123, 893-900. [CrossRef]

18. Feiner, D.M.; Griffin, J.H. A fundamental model of mistuning for a single family of modes / / ASME Turbo Expo 2002: Power for Land, Sea, and Air. Am. Soc. Mech. Eng. 2002, 124, 953-964.

19. Lim, S.H.; Bladh, R.; Castanier, M.P.; Pierre, C. Compact, generalized component mode mistuning representation for modeling bladed disk vibration. AIAA J. 2007, 45, 2285-2298. [CrossRef]

20. Hurty, W.C. Vibrations of structural systems by component mode synthesis. J. Eng. Mech. Div. 1960, 86, $51-70$.

21. Hurty, W.C. Dynamic analysis of structural systems using component modes. AIAA J. 1965, 3, 678-685. [CrossRef]

22. Benfield, W.A.; Hruda, R.F. Vibration analysis of structures by component mode substitution. AIAA J. 1971, 9, 1255-1261. [CrossRef]

23. MacNeal, R.H. A hybrid method of component mode synthesis. Comput. Struct. 1971, 1, 581-601. [CrossRef]

24. Suarez, L.E.; Singh, M.P. Improved fixed interface method for modal synthesis. AIAA J. 1992, 30, $2952-2958$. [CrossRef]

25. Bai, B.; Bai, G.; Li, C. Application of improved hybrid interface substructural component modal synthesis method in vibration characteristics of mistuned blisk. Chin. J. Mech. Eng. 2014, 27, 1219-1231. [CrossRef]

26. Johnson, C.D.; Kienholz, D.A. Finite element prediction of damping in structures with constrained viscoelastic layers. AIAA J. 1982, 20, 1284-1290.

27. Zheng, H.; Tan, X.M.; Cai, C. Damping analysis of beams covered with multiple PCLD patches. Int. J. Mech. Sci. 2006, 48, 1371-1383. [CrossRef] 
28. Sinha, A. Reduced-order model of a bladed rotor with geometric mistuning. J. Turbomach. 2009, 131, 031007. [CrossRef]

29. Curà, F.; Mura, A.; Scarpa, F. Modal strain energy based methods for the analysis of complex patterned free layer damped plates. J. Vib. Control 2012, 18, 1291-1302. [CrossRef]

30. Yoon, Y.C.; Kim, K.H.; Lee, S.H. Dynamic particle difference method for the analysis of proportionally damped system and cracked concrete beam. Int. J. Fract. 2017, 203, 237-262. [CrossRef]

31. Sun, W.; Wang, Z.; Zhu, M.; Du, G. Identifying the mechanical parameters of hard coating with strain dependent characteristic by an inverse method. Shock Vib. 2015, 2015, 487457. [CrossRef]

32. Chandrashaker, A.; Adhikari, S.; Friswell, M.I. Quantification of vibration localization in periodic structures. J. Vib. Acoust. 2016, 138, 021002. [CrossRef]

33. Prudhomme, C.A.; Holtzinger, J.; Goldstein, G.H.; Tzivanis, M.J.; Noonan, W.E.; Austin, R.J. Thermal Spray Masking Tape. U.S. Patent 9,434,137, 6 September 2016.

(c) 2017 by the authors. Licensee MDPI, Basel, Switzerland. This article is an open access article distributed under the terms and conditions of the Creative Commons Attribution (CC BY) license (http://creativecommons.org/licenses/by/4.0/). 